\title{
Modeling of Diffusion Transport through Oral Biofilms with the Inverse Problem Method
}

\author{
Rui Ma ${ }^{1 \dagger}$, Jie Liu ${ }^{2 \dagger}$, Yun-tao Jiang ${ }^{1}$, Zheng Liu ${ }^{1}$, Zi-sheng Tang ${ }^{1}$, Dong-xia Ye ${ }^{1}$, Jin Zeng ${ }^{2 \star}$, Zheng-wei Huang ${ }^{1 *}$ \\ ${ }^{1}$ Department of Endodontics, Ninth People's Hospital, Shanghai Jiao Tong University School of Medicine, Shanghai Key \\ Laboratory of Stomatology, Shanghai, China \\ ${ }^{2}$ Department of Mathematics, Shanghai Jiao Tong University, Shanghai, China
}

\begin{abstract}
Aim The purpose of this study was to develop a mathematical model to quantitatively describe the passive transport of macromolecules within dental biofilms.

Methodology Fluorescently labeled dextrans with different molecular mass (3 kD, $10 \mathrm{kD}, 40 \mathrm{kD}, 70 \mathrm{kD}, 2000 \mathrm{kD})$ were used as a series of diffusion probes. Streptococcus mutans, Streptococcus sanguinis, Actinomyces naeslundii and Fusobacterium nucleatum were used as inocula for biofilm formation. The diffusion processes of different probes through the in vitro biofilm were recorded with a confocal laser microscope.
\end{abstract}

Results Mathematical function of biofilm penetration was constructed on the basis of the inverse problem method. Based on this function, not only the relationship between average concentration of steady-state and molecule weights can be analyzed, but also that between penetrative time and molecule weights.

Conclusion This can be used to predict the effective concentration and the penetrative time of anti-biofilm medicines that can diffuse through oral biofilm. Furthermore, an improved model for large molecule is proposed by considering the exchange time at the upper boundary of the dental biofilm.

Keywords oral biofilm, diffusion model, boundary condition, inverse problem method

Received Jul. 31, 2010; Revision accepted Nov. 18, 2010

\section{Introduction}

Microorganisms are traditionally studied, characterized and identified as planktonic, single-cell creatures. However, detailed studies of bacterial communities in different environments have lead to the conclusion that planktonic growth rarely exists for microorganisms in nature (Costerton $e t$ al., 1995). Generally, bacteria colonize in the form of persistent, surfaces-dependent and matrixencapsulated communities, referred to as biofilms. Since the investigation of microbial aggregates on tooth surfaces by Antonie van Leeuwenhoek, it has been well documented that oral biofilms are responsible for two common human oral diseases: dental caries (tooth decay) and periodontitis (gum disease).
The ability of bacteria to attach on surfaces and form biofilms can bring them many advantages over their planktonic relatives (Rahman et al., 2009). Biofilms have been found to protect the microbial community from environmental stresses, and to shelter bacteria from the immune response and antibiotic penetration (Watson et al., 2005). Most of these protection characters of biofilm can be explained by invoking the phenomenon of diffusion (Gomes et al., 2009).

Diffusion is the process by which the matter is transported from one part of a system to another as a result of random molecular motions (Crank, 1975). The diffusive properties of macromolecules are critical aspects of the growth and pathogenicity of biofilms. The diffusion process of biofilm can directly impact the antibody access to the biofilm 
(Anderl et al., 2000), the penetration of medicines into biofilms (Douglas, 2009), transfer of extracellular DNA (Perry et al., 2009) and the intercellular signals for bacterial communication (Davies et al., 1998; Huang et al., 2009). It also influences nutrient delivery into biofilms in the form of polysaccharides and proteins (Takenaka et al., 2009).

Although much research has been carried out on diffusion in biofilms (Bryers and Drummond, 1998; Nicholson and Tao, 1993; Thurnheer et al., 2003; Rani et al., 2005; Takenaka et al., 2009), there is little consensus on the estimated diffusion coefficients. The purpose of this study was to use time-lapse microscopy to observe the dynamic process of diffusion through the biofilm, on the base of which one could construct the diffusion model to simulate the whole process, and then use the inverse problem method to calibrate the mathematic simulation results with the real diffusion process. These research data can help to analyze the diffusion process, and can be used to predict the biofilm diffusion for the guidance of treatments against biofilm diseases.

\section{Materials and Methods}

\section{Bacterial strains and culture media}

Four strains of oral colonizers, namely Streptococcus mutans UA159, Streptococcus sanguinis 10557, Actinomyces naeslundii ATCC 12104 and Fusobacterium nucleatum ATCC 25586, were used as inocula for biofilm formation. Among these four strains, Streptococcus mutans and Streptococcus sanguinis are pioneer colonizers of oral biofilm, while Actinomyces naeslundii and Fusobacterium nucleatum serve as the frame of biofilm and contribute to the heterogeneity of biofilm. For each species, the optical density of the culture medium was adjusted to 1.0 at $550 \mathrm{~nm}$. Biofilms were formed on glass slides, which were pre-coated with saliva, in 6-well polystyrene cell culture plates, as described previously (Guggenheim et al., 2004) with minor modifications. Wells were inoculated with mixed cell suspensions $(200 \mu \mathrm{L})$ prepared from equal volumes of culture media containing the various species, and incu- bated anaerobically at $37^{\circ} \mathrm{C}$. BM-5 was used as the universal medium (Hodgson et al., 2001) for biofilm, and was replenished by aspirating the spent medium and adding fresh medium every 24 hours. After 72 hours, the glass slides with biofilms were removed for observation.

\section{Biofilm staining and fluorescent mass trans- port probes}

Extracellular polymeric substances (EPS) were stained by incubating biofilms for 20 minutes at $4^{\circ} \mathrm{C}$ with $0.2 \mathrm{mg} \cdot \mathrm{mL}^{-1}$ Concanavalin A fluorescein conjugate (Molecular Probes, product no. C827, Eugene, Oregon, USA) according to the manufacturer's instructions. The following fluorescent probes were prepared at room temperature: $0.2 \mathrm{mg} \cdot \mathrm{mL}^{-1}$ tetramethylrhodamine-conjugated dextrans (TMR-dextran; Molecular Probes, Eugene, Oregon, USA) with molecular mass of 3000 (3 KD-Dex, product no. D3307), 10000 (10 KD-Dex, product no. D1816), 40000 (40 KD-Dex, product no. D1842), 70000 (70 KD-Dex, product no. D1819) and 2000000 (2 $000 \mathrm{KD}-\mathrm{Dex}$, product no. D7139) according to the manufacturer's instructions.

\section{Microscopy and image analysis}

Stained biofilms were examined using a DMIRE2 inverted microscope (Leica Mikroskopie $\mathrm{GmbH}$, Wetzlar, Germany) with a UV laser (Coherent Inc., Santa Clara, USA), a He-Ne laser (Uniphase Vertriebs GmbH, Eching/Munich, Germany), an Ar laser (Coherent Inc.), and a TCS SP2 computeroperated confocal laser scanning system (Leica Lasertechnik GmbH, Heidelberg, Germany). Filters were set to 494 to $518 \mathrm{~nm}$ for detection of Concanavalin A, and 555 to $580 \mathrm{~nm}$ for TMR. Confocal images were obtained by using a $20 \times$ lens (numerical aperture, 0.7; zoom factor, 5.988). Image acquisition was done in the xzt scan mode that recorded a vertical section (xz-section) of the biofilm many successive times. The scan speed was 1000 image lines per second, providing a series of scan photos with $\Delta t$ at $823 \mathrm{~ms}$. Shortly after the continuous scan was started, $20 \mu \mathrm{L}$ of fluorescent mass transport probe was placed at the center of the visual field. The diffusion data 
were processed on a Silicon Graphics 320 visual workstation (Mountain View, USA), which yields a value called the fluorescent intensity index (FII). This index provides a profile consistent with the quantity of diffusion probes entering the biofilm. Five experiments for different probes respectively, with three repeats each, were performed to collect the diffusion data.

\section{Modeling of diffusion through biofilm}

The mathematical theory of diffusion is based on the hypothesis that the rate of transfer of diffusing substance through unit area of a section is proportional to the concentration gradient measured normal to the section. This is described by Fick's law of diffusion

$$
F=-D \frac{\partial C}{\partial x}
$$

where $F$ is the rate of transfer per unit area of section, $C$ is the concentration of the diffusing substance, $x$ is the space coordinate measured normal to the section, and $D$ is the diffusion coefficient.

Fick's law describes the steady state of diffusion. The fundamental differential equation of diffusion is derived from Equation 1 and the conservation of mass principle, which is the most basic principle for all quantitative models,

$$
\frac{\partial C}{\partial t}-\operatorname{div}(D \operatorname{grad} C)=0
$$

If $D$ is taken as a constant (de Beer et al. 1997), in a single dimension, the process of molecules passing through oral biofilm can be given by

$$
\frac{\partial C}{\partial t}-\frac{\partial}{\partial x}\left(D \frac{\partial c}{\partial x}\right)=0
$$

where $x$ is the vertical distance, $t$ is the time, and $C(x, t)$ is the concentration of molecules at distance $x$ from the upper surface at time $t$.

If the size of the object studied is large, and what we wish to know is the situation in the small range inside the object during a very short period of time, the effect of the boundary condition can be ignored (Popov and Poo, 1992). As an approximation, this object can be considered to fill up the entire space. Consequently, the problem can be transformed as an initial value problem (or
Cauchy problem). Many previous results were based on this hypothesis (Birmingham et al., 1995; Bryers and Drummond, 1998; Nicholson and Tao, 1993; Thurnheer et al., 2003). The experimental biofilm, however, is very thin. The diffusion process through the biofilm is very quick (Rani et al. 2005). In our experiment, the biofilm is only a few micrometers thick. It takes a very short period of time (less than 2 second) for molecules to reach the lower boundary of the oral biofilm. So the Cauchy condition cannot be applied to solve the current problem, and the initial boundary condition should not be ignored.

The first assumption for the diffusion model is that the concentration is a constant at the upper boundary. This assumption is reasonable because the amount of the fluorescently labeled dextrans above the biofilm is much larger than the amount of which diffuse into the biofilm. Thus, the concentration of the fluorescent material does not change during the diffusion process. This is consistent with our experiment. Therefore, we have

$$
\begin{gathered}
C(l, t)=C_{0} \\
\text { for } 0<t<\infty
\end{gathered}
$$

where $C_{0}$ is concentration of upper boundary and $l$ is the thickness of the oral biofilm. In fact, $C_{0}$ is also the concentration of steady state under the assumption.

Second, because the bottom of the oral biofilm is fixed on a glass slide in the experiments, the fluorescent material cannot diffuse into the glass. The spillover of fluorescence can be assumed to be zero. Thus, the lower boundary condition is

$$
\begin{aligned}
& \frac{\partial C}{\partial x}(0, t)=0 \\
& \text { for } 0<t<\infty
\end{aligned}
$$

As an initial condition, it is clear that the concentration of molecules was zero before diffusion. Therefore, the initial condition is given by

$$
\begin{gathered}
C(x, 0)=0 \\
\text { for } 0<x<l
\end{gathered}
$$

If $C_{0}, D$ is given, then the direct problem (3)-(6) is well-defined, and we have the solution 


$$
C(x, t)=C_{0}-\frac{4 C_{0}}{\pi} \sum_{k=0}^{\infty} \frac{(-1)^{k}}{2 k+1} e^{-\frac{(2 k+1)^{2} \pi^{2} D}{4 l^{2}} t} \cos \frac{(2 k+1) \pi x}{2 l}
$$

Now there is only the information that is observed in the solution procedure, not the diffusion coefficient. We need to seek both the function $C(x, t)$ and $D(C(x, t))$ for the initial boundary value problem, (3)-(6), and the known resulting concentration.

$$
\begin{aligned}
& \int_{0}^{l} C(x, t) \mathrm{d} x=h(t) \\
& \text { for } 0 \leq t \leq T \text { and } 0 \leq x \leq l
\end{aligned}
$$

where $h(t)$ is additional measurement data. In this experiment, $h(t)$ is the expectation of the fluorescent intensity. The concentration cannot be obtained directly in the experiment. The fluorescent intensity is, however, proportional to the diffusion concentration.

This is an inverse problem. Generally, it is not possible to find a function $C(x, t)$ such that all of the conditions (4)-(6) (8) are satisfied. Then the problem should be proposed as

$$
J(D(C))=\inf \left(\left\|\int_{0}^{l} C(x, t) \mathrm{d} x-h(t)\right\|\right)
$$

where $C(x, t)$ satisfies Equations 3-6. Sometimes the inverse problem is not posed properly, and it requires regularization techniques to find an acceptable solution.

\section{Results and Discussion}

Images of the penetration of $10 \mathrm{KD}$-Dex into artificial supragingival plaque as a function of time are shown in Figure 1. The biofilm thickness at $72 \mathrm{~h}$ was $24.7 \pm 8.0 \mu \mathrm{m}$ (mean and standard deviation). The probe was placed on the biofilm shortly after the continuous scan of the vertical section (xz-section) of the biofilm. As seen from the time series recording, the probe progressively migrated to greater depths with longer incubation periods, until it was distributed throughout much of the volume of the biofilm.

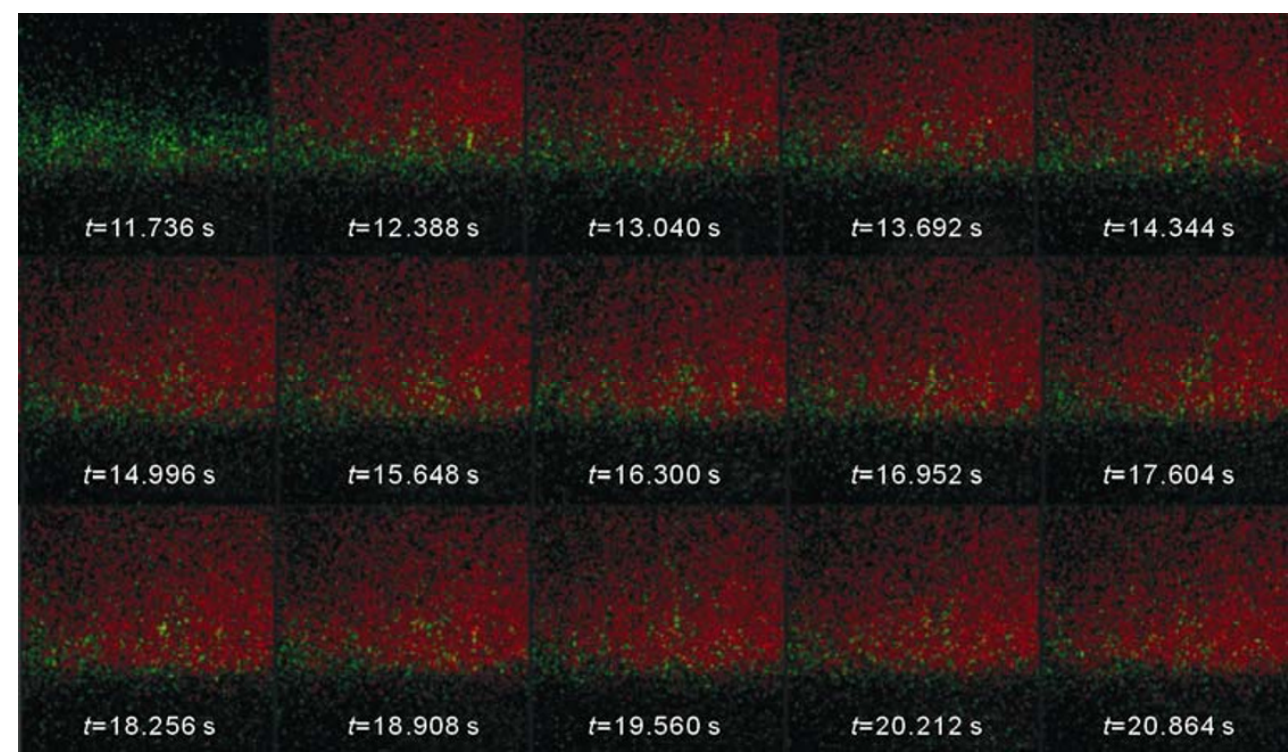

Figure 1 CLSM images of vertical sections (xz sections) of 72-h biofilms, showing the diffusion of 10 KD-Dex as a function of time. Biofilm EPS was stained with Concanavalin (green). The mass transport probe was conjugated to Tetramethylrhodamine (red). Images were obtained with a $20 \times$ objective, with a time delay $(\Delta t)$ of $823 \mathrm{~ms}$

\section{Computing results of the diffusion model}

The data of $3 \mathrm{kD}, 10 \mathrm{kD}, 70 \mathrm{kD}$ and $2000 \mathrm{kD}$ was used to construct the diffusion model. That of $40 \mathrm{kD}$ will be used later for verification.
Figure 2 illustrates the change in the fluorescent intensity with time for macromolecules with different molecular mass. The diffusion process simulated by the results of our model is quite similar to the actual diffusion process. Table 1 
gives diffusion coefficients of the process determined by the model. The error analysis and the modifications will be presented in the next section.

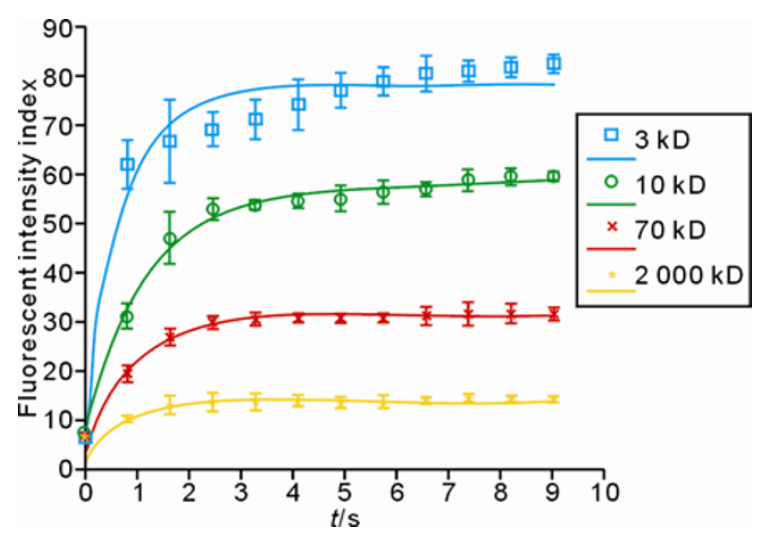

Figure 2 Mean fluorescent intensity index of the diffusing substances with time, for macromolecules with different molecular sizes

From these diffusion equations of different probes indicated in Figure 2, we can obtain the relation between average concentration of steadystate and molecule weights, and the relation between penetrative time and molecule weights.

The relationship between molecular mass and the average steady-state fluorescent intensity index that diffuse through the biofilm are indicated in Figure $3 \mathrm{~A}$. The smaller the weight of the molecules, the more they can diffuse into the biofilm. Furthermore, they satisfy the following formula:

$$
C=14.25+62.24 \times e^{-0.02 x}
$$

here $C$ is the average fluorescent intensity and $x$ is the molecular mass. Then we can predict the average concentration of the fluorescently labeled dextrans in the biofilm if the molecular mass is given. For verification, we calculate the rate of the average concentration of $40 \mathrm{kD}$ according to Equation 10. The numerical result is 41.157. Meanwhile, the result of the experiment is 40.645 , which means the relative error is only $1.3 \%$. For practical use, the effective concentration of biofilm control medicines within biofilms can be predicted based on this mathematical model. This can be of help for clinicians to decide the dose of those medicines applied.

Table 1 Diffusion coefficients and mean square error of computation determined by the model

\begin{tabular}{lrrrr}
\hline Molecular weights $(\mathrm{kD})$ & 3 & 10 & 70 & 2000 \\
\hline Fluorescent intensity index of steady state $(\mathrm{FII})$ & 77.89 & 57.95 & 31.30 & 14.05 \\
Diffusion coefficients / $\left(\mu \mathrm{m} \cdot \mathrm{s}^{-2}\right)$ & 74.79 & 12.69 & 13.08 & 19.95 \\
Mean square error & 3.98 & 2.01 & 1.84 & 1.72 \\
\hline
\end{tabular}
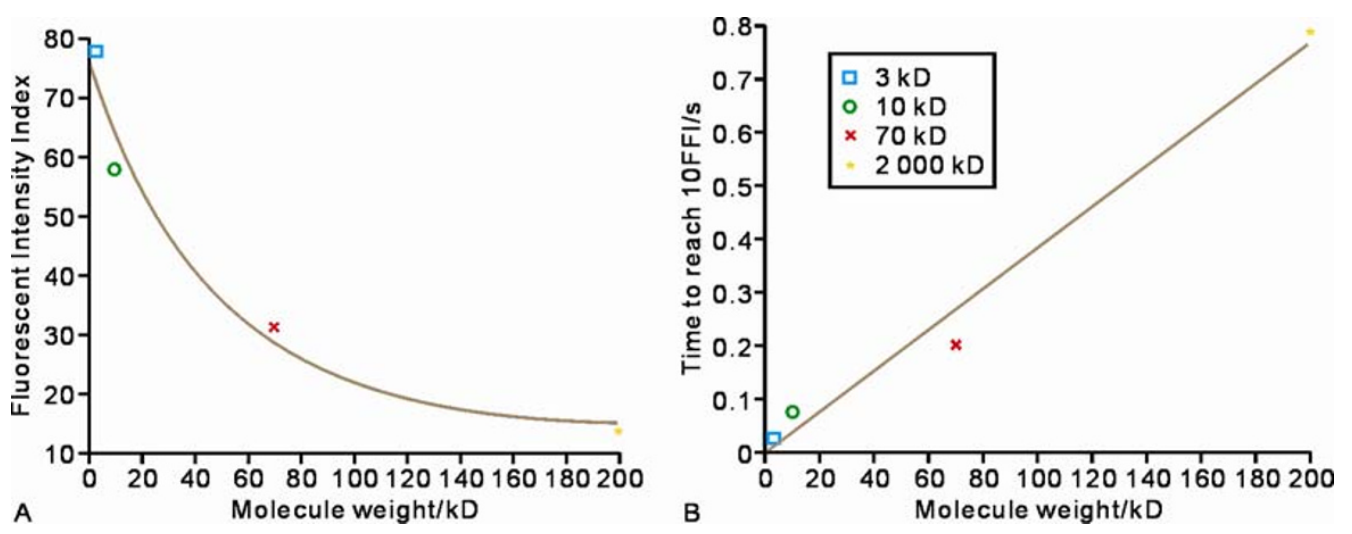

Figure 3 The mean fluorescent intensity of steady-state (A) and the time (B) for macromolecules' fluorescent intensity index to reach 10 FIIs as a function of molecular mass

Figure 3B illustrates the diffusion speed of molecules of different molecular mass. The relation between the time to reach 10 FIIs and the molecular mass is approximately linear and mono- tone increasing. This relation can be expressed as:

$$
t=0.0038 x+0.0012
$$


here $t$ is the time that the fluorescent intensity reach 10 FIIs and $x$ is the molecular mass. It means the lower the molecular mass, the faster they diffuse through the biofilm. For example, for $70 \mathrm{kD}$ molecules, it takes about $0.2 \mathrm{~s}$ to reach 10 , but for $2000 \mathrm{kD}$, the time is around $0.78 \mathrm{~s}$. This relation will help us to know the penetrative time of anti-biofilm medicines that diffuse through oral biofilm and could be used to predict the time-lapse diffusion effect of medicines to diffuse through the biofilm.

By analyzing the error between the simulation results and the experiment data for every point (Figure 2), it can be found that the error of the earlier time point is greater than those of the later, especially for the larger probes. Analysis of these characteristic leads to the following explanation: The concentration of molecules at the upper surface of the oral biofilm is assumed to be constant in the model above. This assumption is reasonable when the fluorescent molecules enter the biofilm very quickly, such as the $3 \mathrm{kD}$ dextran. The larger molecules, such as $70 \mathrm{kD}$ and $2000 \mathrm{kD}$ dextrans, need more time to enter the plaque. In this situation, the error of the early points will be greater than those of the later points if the starting position is considered as constant and the time for entering the biofilm can be ignored. Based on this explanation, we propose the improved method in the following subsections.

\section{The influences of upper boundary condition}

If the entry of molecules at the upper surface takes some time, we may assume that it satisfies Newton's law. This means that the diffusion rate at the boundary is proportional to the difference in concentration of the media. Thus, the corresponding boundary condition can be written as

$$
\begin{gathered}
\frac{\partial C}{\partial x}(l, t)=L\left[C_{0}-C(l, t)\right] \\
\text { for } 0<t<\infty
\end{gathered}
$$

where $L$ is the exchange coefficient.

The solution of Equation 3 at the condition of $(4,5,11)$ is:

$$
C(x, t)=C_{0}\left(1+\sum_{k=1}^{\infty} \frac{2 L l \sqrt{(L l)^{2}+\mu_{n}^{2}}}{\mu_{n}\left[(L l)^{2}+L l+\mu_{n}^{2}\right]} \mathrm{e}^{-\frac{\mu_{n}^{2} D}{l} t} \cos \frac{\mu_{n} x}{l}\right.
$$

where the $\mu_{n}$ 's are the non-zero positive roots of the following equation

$$
\operatorname{ctg} u=\frac{1}{L l} u
$$

Table 2 gives the relative error of the two models. It can be found that the errors of computational results are lower when the exchange coefficient is added into the model for $10 \mathrm{kD}$, $70 \mathrm{kD}$, and $2000 \mathrm{kD}$ dextrans. In particular, for $70 \mathrm{kD}$ dextran, the mean square error is reduced from 1.84 to 0.43 .

For $3 \mathrm{kD}$ dextran, however, the mean square error increases a little when the exchange coefficient is added into the model. The reason could be that the weight of the molecules is very small, thus the exchange at the boundary is fast. In this situation, it is more reasonable to assume that the exchange takes place immediately after diffusion, and that the concentration of molecules at the upper surface of the oral biofilm is constant instead of being subjected to exchange according to Newton's law.

Table 2 Mean square error of two models

\begin{tabular}{lcccc}
\hline Molecular weights (kD) & 3 & 10 & 70 & 2000 \\
\hline Mean square error of initial model & 3.98 & 2.01 & 1.84 & 1.72 \\
Mean square error of improved model & 4.20 & 1.37 & 0.43 & 0.71 \\
\hline
\end{tabular}

\section{Conclusion}

In this paper, the diffusion model is reconstructed with experimental data by inverse problem methodology. According to the actual physical back- ground, we use Partial Differential Equation with initial-boundary conditions to describe the diffusion of the fluorescent molecules. As the actual diffusion process is taking place on a thin biofilm, and the entire process is almost complete within 2 seconds, the reasonable boundary conditions are critically 
important to the solution of the problem. Thus, the models fully consider the influences of different boundary conditions on the diffusion process. The model 1 (Equation 7) is satisfied from the point of view of the mean square error. Furthermore, an improvement is made. If we consider the exchange time, the model would be more suitable for larger molecules. Based on this research, we obtained the relation between average concentration of steadystate and molecule weights, and the relation between penetrative time and molecule weights. This mathematic model can be helpful to describe the passive transport of macromolecules within biofilms, which would be useful for the prediction of the effective concentration of antimicrobial medicines to diffuse through oral biofilms in the future.

\section{Acknowledgements}

This work was supported by a grant from the National Natural Science Foundation of China (NSFC) No. 81070826/30872886/30400497, and partly Sponsored by Shanghai Rising-Star Program No. 09QA1403700. The computational work was partially funded by Shanghai Leading Academic Discipline Project (Project Number: S30206) and the Science and Technology Commission of Shanghai (08DZ2271100).

\section{References}

Anderl JN, Franklin MJ, Stewart PS (2000). Role of antibiotic penetration limitation in Klebsiella pneumoniae biofilm resistance to ampicillin and ciprofloxacin. Antimicrob Agents Chemother, 44 (7): 18181824.

Birmingham JJ, Hughes NP, Treloar R (1995). Diffusion and binding measurements within oral biofilms using fluorescence photobleaching recovery methods. Philos Trans R Soc Lond B Biol Sci, 350(1334): 325-343.

Bryers JD, Drummond F (1998). Local macromolecule diffusion coefficients in structurally non-uniform bacterial biofilms using fluorescence recovery after photobleaching (FRAP). Biotechnol Bioeng, 60(4): 462-473.

Costerton JW, Lewandowski Z, Caldwell DE, Korber DR, Lappin-Scott HM (1995). Microbial biofilms. Annu
Rev Microbiol, 49: 711-745.

Crank J (1975). The mathematics of diffusion. 2nd ed. New York: Oxford university press.

Davies DG, Parsek MR, Pearson JP, Iglewski BH, Costerton JW, Greenberg EP (1998). The involvement of cell-to-cell signals in the development of a bacterial biofilm. Science, 280 (5361): 295-298.

de Beer D, Stoodley P, Lewandowski Z (1997). Measurement of local diffusion coefficients in biofilms by microinjection and confocal microscopy. Biotechnol Bioeng, 53(2): 151-158.

Douglas LJ (2009). Penetration of antifungal agents through Candida biofilms. Methods Mol Biol 499: $37-44$.

Gomes FI, Teixeira P, Azeredo J, Oliveira R (2009). Effect of farnesol on planktonic and biofilm cells of Staphylococcus epidermidis. Curr Microbiol, 59(2): 118122.

Guggenheim B, Guggenheim M, Gmür R, Giertsen E, Thurnheer T (2004). Application of the Zürich biofilm model to problems of cariology. Caries Res, 38(3): 212-222.

Hodgson RJ, Lynch RJ, Watson GK, Labarbe R, Treloar $\mathrm{R}$, Allison C (2001). A continuous culture biofilm model of cariogenic responses. J Appl Microbiol, 90 (3): 440-448.

Huang Z, Meric G, Liu Z, Ma R, Tang Z, Lejeune P (2009). luxS-based quorum-sensing signaling affects Biofilm formation in Streptococcus mutans. $J \mathrm{Mol}$ Microbiol Biotechnol, 17 (1): 12-19.

Khabibor Rahman N, Bakar MZ, Hekarl Uzir M, Harun Kamaruddin A (2009). Modelling on the effect of diffusive and convective substrate transport for biofilm Math Biosci, 218(2): 130-137.

Kubota H, Senda S, Nomura N, Tokuda H, Uchiyama H (2008). Biofilm formation by lactic acid bacteria and resistance to environmental stress. $J$ Biosci Bioeng, 106(4): 381-386.

Nicholson C, Tao L (1993). Hindered diffusion of high molecular weight compounds in brain extracellular microenvironment measured with integrative optical imaging. Biophys $J, 65(6)$ : 2277-2290.

Perry JA, Cvitkovitch DG, Lévesque CM (2009). Cell death in Streptococcus mutans biofilms: a link between CSP and extracellular DNA. FEMS Microbiol Lett, 299(2): 261-266.

Popov S, Poo MM (1992). Diffusional transport of macromolecules in developing nerve processes. $J$ Neurosci, 12(1): 77-85. 
Rani SA, Pitts B, Stewart PS (2005). Rapid diffusion of fluorescent tracers into Staphylococcus epidermidis biofilms visualized by time lapse microscopy. Antimicrob Agents Chemother, 49(2): 728-732.

Takenaka S, Pitts B, Trivedi HM, Stewart PS (2009). Diffusion of macromolecules in model oral biofilms. Appl Environ Microbiol, 75 (6): 1750-1753.

Thurnheer T, Gmür R, Shapiro S, Guggenheim B (2003).
Mass transport of macromolecules within an in vitro model of supragingival plaque. Appl Environ Microbiol, 69(3): 1702-1709.

Watson PS, Pontefract HA, Devine DA, Shore RC, Nattress BR, Kirkham J et al. (2005). Penetration of fluoride into natural plaque biofilms. $J$ Dent Res, 84(5): 451-455.

${ }^{\dagger}$ These authors contributed equally to this work.

*Corresponding authors: Zhengwei Huang and Jin Zeng

Zhengwei Huang

Address: Department of Endodontics, Ninth People's Hospital, Shanghai Jiao Tong University School of Medicine, No 639 Zhizaoju Road, Shanghai 200011, China

Tel: 8613764248949 Fax: 862163135412 E-mail: huangzhengwei@shsmu.edu.cn

Jin Zeng

Address: Department of Mathematics, Shanghai Jiao Tong University, No 800 Dongchuan Road, Shanghai 200240, China

Tel: 8613817096145 Fax: 862154743152 E-mail: zengjin@sjtu.edu.cn 